Cardiol Young 2000; 10: 176

(C) Greenwich Medical Media Ltd. ISSN 1047-9511

\title{
Diary of Events
}

\section{0-31 March 2000}

XXI Séminaire de Cardiologie Pédiatrique: Gènes, syndromes, séquences et tout ça. Paris, France.

Enquiries: Secrétariat du Service de Cardiologie Pédiatrique, Hospital Necker/Enfants-Malades, 149 rue de Sèvres, 75743 Paris cedex 15, France. Fax: (+33) 144494340

\section{5-7 April 2000}

$10^{\text {th }}$ Charleston Symposium on Congenital Heart Disease, Charleston, South Carolina, USA.

Enquiries: J Philip Saul, MD, Medical Unıversity of South Carolina, The Children's Heart Center, 165 Ashley Ave, PO Box 250915, Charleston, SC 29425, USA. Fax: (+843) 792 3284

\section{7-20 April 2000}

$9^{\text {th }}$ Annual Symposium "Cardiology in the Young", London. Enquiries: Courses \& Conferences Office, Institute of Child Health, 30 Guilford St, London WC1N 1EH, UK. Tel: +44 (0)20 7829 8692; Fax: +44 (0)20 7831 6902; e-mail: Courses@ich.ucl.ac.uk

\section{8-20 May 2000}

International Symposium on The Developing Heart, Prague. Enquiries: Czech Medical Association J.E. Purkyne, PO Box 88, Sokolská 31, 12026 Prague 2, Czech Republic. Tel: 420 2297 271; Fax: 4202294610 ;

e-mail: senderova@cls.cz; www.biomed.cas.cz/fgu/cardiol/ dh2000.htm

\section{9-20 May 2000}

International Conference "3-Dimensional Imaging of the Heart" the standard for the New Millennium in Pediatric Cardıology and Cardiac Surgery. Venice, May 19-20, 2000. Enquiries: Segreteria Organizzatıva - C.S.S., Viale Mazzini 70, 50131 Firenze, Italy. Tel: 055248 0202; Fax: 055248 0246; e-mail: css@css-congressi.it

\section{4-17 June 2000}

$35^{\text {th }}$ Annual Meeting Association for European Paediatric Cardiology, Strasbourg, France, 14-17 June 2000. Enquiries: Dr Bernard de Geeter, Cardiologie Pédiatrıque et Ftale, 3 rue Simonis, F-67100 Strasbourg. Fax: +33 (388) 3430 04; email: drdegeeter $@$ mail.sdv.fr

12th International Congress "Cardiotim", Nice, June 14-17, 2000. Enquiries: Cardiotım 2000/Miller Freeman, 70 rue Rivay, 92532 Levallors-Perret Cedex, France. Tel $+33(0) 147$ 6651 96; Fax:+33(0)1 47562110 ; e-mail: info@cardiotim.asso.fr; www.cardiotim.asso.fr

\section{9-30 June 2000}

New Horizons in Fetal Cardiology, London.

Enquiries: Courses \& Conferences Office, Institute of Child
Health, 30 Gulford St, London WC1N 1EH, UK. Tel: +44 (0)20 7829 8692; Fax: +44 (0)20 7831 6902; e-mall: Courses@ich.ucl.ac.uk

\section{3-14 July 2000}

Advanced Course in Cardiac Morphology, London.

Enquiries: Courses \& Conferences Office, Institute of Child Health, 30 Guilford St, London WC1N 1EH, UK. Tel: +44 (0)20 7829 8692; Fax: +44 (0)207831 6902; e-mall: mailto:Courses@ich.ucl.ac.uk Courses@ich.ucl.ac.uk

\section{3-25 August 2000}

International Symposium Current Controversies and Technique in Congenital Heart Surgery. University Hospital, Utrecht, August 23-25, 2000. Enquiries: Mediscon, Organisation or Medical Congresses, PO Box 113, 5660 AC Geldrop, The Netherlands. Tel: +31 (0) 4028522 12; Fax: +31 (0) 402851966 , E-mail: mediscon@iaehv.nl

\section{6-30 August 2000}

22nd Congress of the European Society of Cardiology, Amsterdam, August 26-30, 2000. Enquiries: ECOR - The European Heart House, 2035 Route des Colles, Les Templiers, BP 179, 06903 Sophia Antipolis Cedex, France. Tel: +33 (0)492947600; Fax: + $33(00492947601$. Email: webmaster $(a$ escardio.org

\section{4-7 October 2000}

30 Congresso della Società Italiana di Cardiologia Pedıatrica. Torino, October 4-7, 2000. Enquiries: Segreterta Organizzativa - C.S.C., Vıale Mazzıni 70, 50132 Fırenze, Italy. Tel: 055248 0202; Fax: 055248 0246; e-ma1: css@css-congressi.tt

\section{2-15 November 2000}

$73^{\text {rd }}$ Scientific Session of American Heart Association, New Orleans, LA, USA.

Enquiries: AHA, Scientific Meetıngs, 7272 Greenville Ave, Dallas, TX 75231-4596, USA. Fax: (+214) 373 3406; E-mail: scientificconferences@heart.org; www.americanheart.org/scien tific/confer/index.html

27-31 May 2001

$3^{\text {rd }}$ World Congress of Paediatric Cardiology \& Cardiovascular Surgery, Toronto, Canada, May 27-31 2001. Enquiries: PCCS 2001, c/o Congress Canada, 49 Bathurst Street, Ste.100, Toronto, ON, M5V 2P2, Canada. Tel: +1416-504-4500; Fax: +1-416-504-4505; E-mail: congca@icongresscan.com 